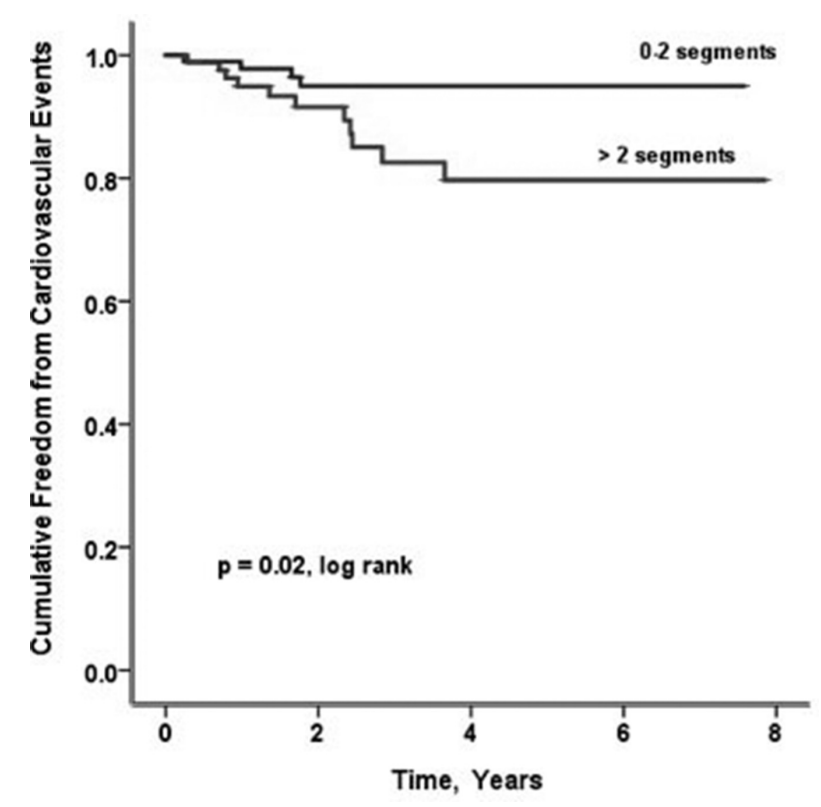

Abstract 115 Figure 1

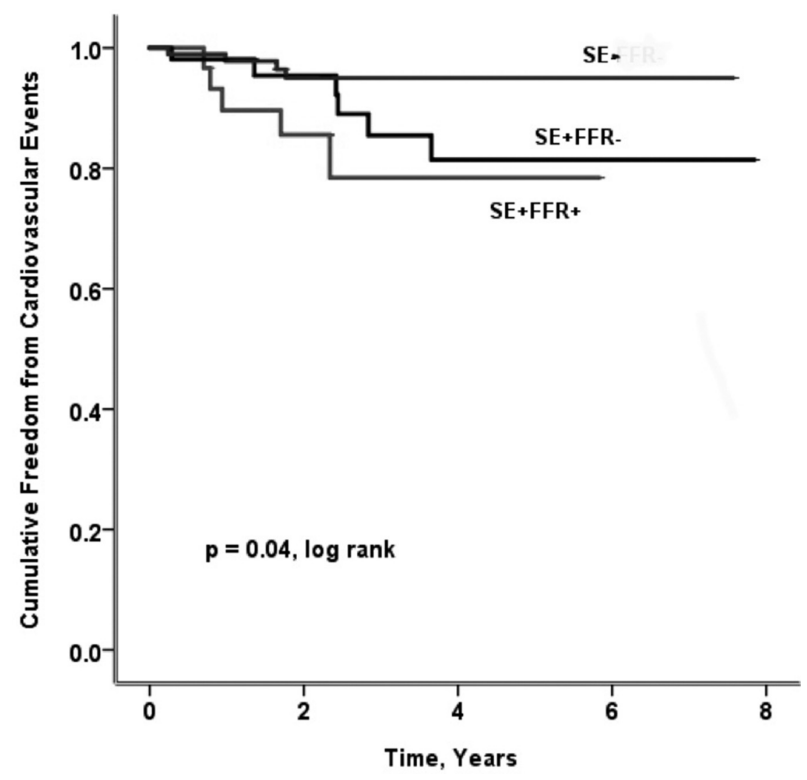

Abstract 115 Figure 2

Conclusion In a patient population with significant CV risk factors, a normal SE effectively ruled out abnormal FFR. The greatest discordance was seen in patients with abnormal SE/ normal FFR. In this group, patients had similar outcomes compared to those with abnormal SE/positive FFR but worse outcomes compared to patients with a normal SE. These findings have significant clinical implications.

\section{CT CORONARY ANGIOGRAPHY VERSUS CORONARY ARTERY CALCIUM SCORING FOR THE OCCUPATIONAL ASSESSMENT OF MILITARY AIRCREW}

${ }^{1}$ lain Parsons*, 'Christopher Pavitt, ${ }^{2}$ Rebecca Chamley, ${ }^{3}$ Jo D'Arcy, Ed Nicol. 'Royal Brompton; ${ }^{2}$ Royal Berkshire; ${ }^{3}$ John Raddliffe

\subsection{6/heartjnl-2017-311726.115}

Introduction To ensure flight safety military aircrew undergo regular clinical and occupational assessment. Coronary artery calcium scoring (CACS) is established as an imaging modality to non-invasively assess coronary artery disease (CAD). CT coronary angiography (CTCA) potentially offers a more accurate assessment of CAD but has not been formally assessed in military aircrew. This retrospective cohort study is designed to compare the theoretical differences in downstream investigations and occupational outcomes in aircrew with suspected CAD comparing CTCA with existing CACS pathways.

Methods A 2 year retrospective cohort study of consecutive UK military patients who underwent a CTCA and CACS. Patient demographics, CTCA and CACS results and initial and final occupational restrictions were analysed comparing current UK, Canadian and US pathways.

Results 44 patients underwent CACS and CTCA. The commonest indication for a CTCA was a positive exercise ECG. Increasing CACS, stenosis severity and stenosis burden were associated with significantly greater likelihood of occupational restriction $(\mathrm{p}=<0.01)$. Following CTCA $(26 / 44,59 \%)$ of patients were found to have evidence of CAD with $(13 / 44,30 \%)$ having at least a single vessel stenosis 50\%. All of these patients had subsequent occupational restrictions. Two patients with a calcium score 10 had at least one single vessel stenosis 50\%.

Conclusion A CTCA pathway is potentially a better discriminator of CAD burden in aircrew when compared with CACS and may reduce downstream testing, allowing a more efficacious approach to CAD assessment in military aircrew.

\section{INCIDENTAL FINDING OF CORONARY ARTERY CALCIFICATION IN NON-TRIGGERED HIGH-RESOLUTION THORACIC COMPUTED TOMOGRAPHY: A RETROSPECTIVE STUDY OF REPORTING STANDARDS IN A SINGLE TRUST}

${ }^{1}$ Sushant Saluja*, 'Janousek Pavel, 'Divya Raj, 'Bruce Irwin, 'David Zhu, 'Timothy Gray, ${ }^{1}$ Mark Hargreaves, ${ }^{1}$ Aung Sett, ${ }^{2}$ Simon Anderson. ${ }^{1}$ Pennine Acute Trust; ${ }^{2}$ Manchester Royal Infirmary

\subsection{6/heartjnl-2017-311726.116}

Aims Coronary artery calcium (CAC) score is an important tool in determining the risk of developing heart disease. The measurement of this score has traditionally been based on using ECG triggered computed tomography (CT). Emerging evidence has revealed that there is excellent concordance between gated and non-gated CT scans in identifying CAC. We aimed to evaluate the incidental prevalence and burden of CAC on non-gated High Resolution CT (HRCT) thorax used 
for patients undergoing lung cancer screening or follow-up for interstitial lung disease.

Methods Computerised Radiology Information Service (CRIS) was manually searched to determine all HRCT scans performed in our Trust from $01 / 05 / 2015$ to $01 / 05 / 2016$. The reports issued by Radiologists and images of selected studies were reviewed.

Results 2152 HRCT scans were performed over this period. Patients were divided into three groups of age $<50$ (Group 1); 50-<60 (Group 2) and 60 (Group 3). 100 scans were randomly selected from each group using a random number generator to give a total of 300 patients. The mean ages of patients in Group 1, 2 and 3 were 42.7 $\pm 2.3,57.4 \pm 1.4$ and $66 \pm 2.7$ respectively. There was, approximately, the same number of males as females in each group. CAC was seen in 10\% of scans in Group 1, 85\% of scans in Group 2\% and 92\% in Group 3. CAC was only reported in 2/10 (20\%) of scans in Group 1, 39/85 (45.9\%) in Group 2 and 41/92 (44.6\%) in Group 3. No scan commented on the absence of calcification. For the positive reports Agatston scores were not calculated and CAC was perceptually graded a mild, moderate or severe. SS and PJ independently calculated the Agatston scores of HRCT scans whose reports did not comment on the degree of calcification (105/187). We excluded 15/105 (14.3\%) scans as they were uninterpretable due to motion artefacts. Of the remaining 90 scans analysed 63/90 (70\%) had severe CAC with an Agatston score of $>400$ with the remaining showing moderate calcification (101-400). Cohen $\hat{\mathrm{I}}^{\circ}$ agreement between the two authors rating was 0.88 (95\% confidence interval [CI] $0.82-0.94)$. Group 2 and 3 had significantly more patients with severe CAC then group $1 \quad(\mathrm{p}<0.001)$. Left anterior descending artery was most commonly affected.

Conclusion This study shows that CAC is under reported on non-gated HRCT scans. Given that respiratory disease is an independent risk factor for developing cardiac disease, we believe that $\mathrm{CAC}$ must be reported on patients having imaging for respiratory conditions. By mentioning the degree of CAC as a standard when reporting non-gated HRCT scans, we can risk-stratify patients and refer them to cardiologists for further investigations, such as stress-echo and commence them on medications for primary prevention of cardiovascular disease. The images of calcified coronary arteries may also potentially have a role in convincing people to make correct lifestyle choices.

\section{APPLYING APPROPRIATE USE CRITERIA FOR TRANSTHORACIC ECHOCARDIOGRAPHY TO CLINICAL PRACTICE}

Zach Robbins*, Malcolm Burgess, Ben Harrison. Aintree University Hospital

\subsection{6/heartjnl-2017-311726.117}

Introduction Transthoracic echocardiography (TTE) is a wellestablished, non-invasive method of investigating multiple pathologies in cardiology and is widely accessible to non-cardiologists. Appropriateness use criteria (AUC) have been developed by the American College of Cardiology Foundation (ACCF), but these are not implemented systematically in everyday practice. Previous studies indicate that approximately 1 in 10 requests may be avoidable. In our hospital the cardiorespiratory department performs over 10,000 TTEs per year. While most requests for TTE are appropriate, a small number of inappropriate requests amounts to an avoidable burden in the workload of department.

Purpose To determine how many inappropriate requests were received by the department and if there were any patterns to the inappropriate requests that would inform the development of screening questions when moving from a paper based to electronic requesting system.

Methods All inpatient and outpatient requests were collected and analysed over 12 consecutive days in January 2015. The system for requesting TTEs was paper based with free text boxes. Requests from primary care were excluded as they had fixed criteria for requesting TTEs. All requests were assigned to the most closely applicable indication and appropriateness score based on the ACCF 2011 criteria. The requesting system was then changed to an electronic system with mandatory screening questions based on the AUC. All inpatient and outpatient requests were again collected over 14 consecutive days, in August/September 2016, once the electronic system was established. Requests were analysed using the same criteria.

Results We analysed 294 requests, 2.4 outpatient requests for each inpatient request, in 2015 and 333 requests, 1.85 outpatient requests for each inpatient request, in 2016. Approximately half of requests came from cardiology services, $47 \%$ in 2015 and $52 \%$ in 2016. The commonest indications were similar both years, with ACCF indications 1 and 2; symptoms (e.g. chest pain, palpitations, dyspnoea, etc.) or test results suggesting cardiac pathology (e.g. abnormal ECG, elevated cardiac biomarkers, etc.) accounting for 22\% of requests in 2015 and $34 \%$ in 2016.

In 2015, 91\% $(n=266)$ of requests were appropriate, 5\% $(n=16)$ of uncertain appropriateness and 4\% were inappropriate $(n=12)$. After implementation of the screening questions through electronic requesting, inappropriate requests had reduced to $1 \%(n=4)$ of total requests, $2 \%(n=5)$ were of uncertain appropriateness and 97\% $(n=324)$ were appropriate $(p=0.0477)$. This equated to an estimated reduction of eight inappropriate or uncertain requests a week.

Conclusion After implementation of screening questions in an electronic requesting system, inappropriate requests fell. Systematic screening of TTE requests can help to reduce inappropriate requests and allow resources to be focused on appropriate indications.

\section{V/Q SINGLE-PHOTON EMISSION COMPUTED TOMOGRAPHY IN NON-THROMBOEMBOLIC PULMONARY ARTERIAL HYPERTENSION}

Kenneth Chan*, Stefanos Ioannidis, John G Coghlan, Margaret Hall, Benjamin E Schreiber. Royal Free Hospital

\subsection{6/heartjnl-2017-311726.118}

Background Pulmonary arterial hypertension (PAH) is a rare cause of pulmonary hypertension (PH) with poor prognosis. Current guidelines recommend ventilation/perfusion (V/Q) scan in screening for thromboembolic cases of $\mathrm{PH}$. We hypothesise that perfusion defects in non-thromboembolic PAH could also render abnormal V/Q scans, and sough to evaluate the incidence and clinical significance of abnormal V/Q SPECT in patients without thromboembolism.

Methods A total of 136 patients underwent V/Q SPECT (single-photon emission computed tomography) and right heart 